\title{
Cars Ecology
}

\section{Cars Ecology}

Fernanda Espinel Fagua9 , Andrés Felipe Garavito Oyola ${ }^{10}$ y Dayanna Alejandra González Sánchez ${ }^{11}$

\section{Resumen}

Este trabajo se centró en la intervención de una estrategia específica que busca ayudar de manera didáctica en el tratamiento para los niños con cáncer. Este es un proyecto que tiene como propósito ayudar a minimizar el dolor psicológico y traumático que portarán estos niños, para cuando acudan a la entidad de salud a realizarse las respectivas quimioterapias. Bragado (2009) menciona que "se ha observado en algunas ocasiones que ante un mismo tipo de quimioterapia algunos pacientes desarrollan náuseas y vómitos intensos. Esto se da por los efectos secundarios de la quimioterapia". La metodología de Cars Ecology cuenta con la realización de carros hechos a base de latas metálicas reusables, que al someterse a una transformación física se puede obtener un nuevo diseño de carro de juguete que es amigable con el medio ambiente y que de igual manera contribuye al entretenimiento del niño o la niña que lo vaya a usar en los servicios donde se realice su tratamiento. Este es un producto garantizado que busca brindar felicidad a los niños al momento de interactuar con el carro ecológico, asegurando acompañamiento en la estadía hospitalaria, dando confianza a sus familiares para la evolución del tratamiento y cumpliendo así nuestro objetivo.

Palabras clave: cáncer, latas metálicas, medio ambiente, depresión, enfermería.

\section{Abstract}

This paper focuses on a specific strategy that seeks to help treat children with cancer didactically. This project aims to help minimize the psychological and traumatic pain these children will suffer when they attend the health center for chemotherapy. The Cars Ecology method involves making toy cars from reusable metal cans after

9 Estudiante, Fundación Universitaria del Área Andina, lespinel6@estudiantes.areandina.edu.co

10 Estudiante, Fundación Universitaria del Área Andina, agaravito4destudiantes.areandina.edu.co

11 Estudiante, Fundación Universitaria del Área Andina, dgonzalez1711estudiantes.areandina.edu.co 
a physical transformation; they are environmentally friendly and keep children entertained while receiving their treatment. This method seeks to bring happiness to children when interacting with toy cars, ensure support during the hospital stay, and give confidence to relatives about treatment evolution.

Keywords: Cancer, Metal cans, Environment, Depression, Nursing.

\section{Introducción}

Dávila (2017) plantea que los niños que tienen cáncer logran tener una problemática muy grande al verse expuestos frente a la depresión. Todo esto se ve reflejado por medio de las terapias y tratamientos. Para ellos esta situación no logra ser nada fácil, y con el paso del tiempo se han venido realizando unos estudios por Shapiro (2002) donde nos indican que la mayoría de las emociones se comienzan a presentar de 6 a 9 meses de edad y esto va evolucionando hasta los 5 años de edad. En la psicología evolutiva se considera que hasta el año y medio los niños y las niñas son capaces de controlar sus emociones, aunque todo esto se ve reflejado dependiendo de la educación que les brindan los padres u otras personas que los rodean, es decir la interacción con la sociedad.

En el artículo de depresión en niños y niñas, el autor González (2006) considera que la depresión sí existe y esta puede manifestarse en los niños y las niñas desde los seis años de edad, pero estas son más intensas, más frecuentes, versátiles y menos reflexivas. En el caso del cáncer infantil, es muy importante reconocer que esta enfermedad puede afectar psicológicamente a los niños y niñas, pero también a sus familiares. Hernández (2006) afirma que la presencia de una enfermedad médica es uno de los principales factores que genera la depresión infantil. Esta enfermedad es muy fuerte no solo en sus tratamientos sino porque, además de esto se genera mucho dolor en el momento de someter al niño a fármacos o quimioterapias.

Calvillo (2004) define que la depresión es un trastorno afectivo, en este se ve implicada la parte emocional, cognitiva, motora y social. Es decir que se presenta un cambio permanente el cual disminuye la capacidad de que los niños con cáncer puedan lograr disfrutar los acontecimientos, pierdan la capacidad de poder comunicarse con los demás y presentar un cambio notorio frente al rendimiento escolar. Esto va acompaña- 
do de algunas acciones y estas pueden ser vistas como conductas de protesta o rebeldía.

Según la psicóloga Silvia Alba, quien investiga sobre la felicidad en los niños con cáncer, afirma que estos cuentan con un $50 \%$ de la felicidad que se debe a los factores genéticos, un $10 \%$ de las experiencias vividas y un $40 \%$ frente a la actividad emocional, que podemos aprender a controlar para ser más felices. Es decir, que la felicidad se aprende. El objetivo de Cars Ecology es que los niños que tienen cáncer puedan usar el $40 \%$ de actividad emocional por medio de un carro, el cual es elaborado con latas de bebidas, con diseños llamativos a la vista de los niños. Esto tiene como énfasis la importancia del impacto ambiental, ya que al momento de reciclar estas latas se disminuye el calentamiento global, mejorando así el medio ambiente.

Pérez, Vélez y Zamora (2015) indican que es un hecho que los niños al ser diagnosticados con cáncer, comienzan a pensar en las terribles consecuencias de su patología y entran en una etapa depresiva que perdurará por todo el transcurso de su tratamiento. Por otro lado, González (2006) comenta que los juguetes contribuyen a disminuir el estrés que pueden causar los tratamientos y quimioterapias, buscando como respuesta a ello un resultado satisfactorio frente a disminuir la depresión infantil Sin embargo, Medina (2019) conduce a que esto se debe a que un juguete es un objeto físico que hace feliz a todo niño, ya que este ayuda a impulsarlos a elevar su imaginación, haciendo a un lado el encierro. Además, un juguete también implica la interacción con otros niños, con la amabilidad, dando a conocer un nuevo entorno social y poder enfrentar los factores de riesgos de esta enfermedad.

\section{Marco teórico}

Los niños que sufren de cáncer son afectados por factores a nivel emocional, psicológico, psicosocial y físicamente, estos son:

Depresión: González (2006) expone que los niños que sufren de algún tipo de cáncer tienden a desarrollar depresión, ya sea por su estadía prolongada en los centros de salud o por el deterioro al que se ven expuestos en su vida cotidiana, pues un niño o niña con cáncer es más vulnerable. El acompañamiento por parte de su familia es indispensable, ya que es importante ser claros con ellos e informarles sobre el procedimiento al que van a someter al niño, teniendo en cuenta también la importancia de brindarles ayudas 
psicológicas. Grau (2002) hace referencia en su artículo a que cuando un niño cae enfermo, toda la familia enferma con él, por lo que los tratamientos deben ser integrales y referidos a todo el núcleo familiar. Por otra parte, González (2006) define que "la depresión es considerada un síndrome que afecta aproximadamente al $15 \%$ y $25 \%$ de los pacientes en general a quienes se les diagnostica de cáncer y a sus familiares".

\section{Factores psicosociales que representa el cáncer y cómo llevarlo}

Bragado (2009) manifiesta que hay algunos chicos que al llegar a padecer cáncer tienden a llegar a afrontar esta enfermedad de buena manera frente al aspecto psicológico sin mostrar cambios a nivel psicológico y emocional, pero no siempre se da de esta manera porque en varios casos al no poder estar en contacto mucho tiempo con espacios externos tienden a estresarse demasiado, notándose en ese punto el desequilibrio frente a sus emociones. Como consecuencia a ello se presentan de una forma más frecuente las disfunciones sociales o el hecho de tener la dificultad de compartir con otras personas. Los niños con cáncer pueden llevar de buena forma esta enfermedad, aunque al decirlo de esta mane- ra no significa que ellos dejen de sufrir, porque en ocasiones son ellos quienes quieren llegar a negar que tienen los síntomas de esta enfermedad, usando un método por medio del cual puedan reprimir sus sentimientos y negar que portan esta enfermedad. Según Roesch (2006) "no hay una estrategia mediante la cual se pueda ayudar a afrontarla, ya que algunos padres, e incluso niños, usaban varios métodos para poder afrontarla sin importar cuál sea la circunstancia”.

Felicidad por medio de un juguete: considerando la depresión en niños con cáncer y el problema psicosocial que puede causar este, es importante contar con diferentes juegos en el hospital, ya que estos ayudan a enfrentar aquellas enfermedades que se consideran complicadas según su ámbito físico y psicológico, para niños menores en un rango de 6 a 9 años de edad. Méndez, Ortigosa y Riquelme (2009) indica que existen tres tipos de juegos los cuales son: juegos terapéuticos, juegos de desarrollo y juegos de distracción. El juego terapéutico se considera como un instrumento que se utiliza para enfrentarse a nuevas experiencias estresantes a las que se somete al niño. Además, a través de esta modalidad de juego se facilita el acceso a la información, la expresión emocional, la comunicación, y la habituación 
al aparataje y situaciones médicas. Esto consiste en darle al niño material médico como mascarillas, jeringuillas, vendas, etc., con el fin de que estos los manipulen como un muñeco, es decir que toman el rol de entrenador y esto facilita el afrontamiento.

Por su parte, Milagros (2018) expresa que es importante considerar que se debe abrir una sala o área de juego con un aspecto lúdico, creativo y sin limitaciones de espacio, brindando comodidad a los niños. Además es importante resaltar que esto aleja el miedo, el estrés y el dolor que genera el ámbito médico. Por otro lado, Pérez y Reyes (2017) manifiesta que por medio del juego se busca que el niño abra un espacio de interacción con los demás niños, ya que esto ayuda a enfrentar las consecuencias y riesgos dependiendo de la enfermedad. Sin embargo hay niños que consideran tener un espacio de juego a solas, ya que hay complicaciones físicas como la movilidad de su cuerpo y estas tienden a hacerlos más delicados. También hay complicaciones psicológicas como la depresión que complica la interacción con otros pacientes, lo que puede retrasar el proceso de afrontamiento, tratamientos y dificultad para la toma de decisiones difíciles.
Segura (2015) expresa que una de las variantes del juego terapéutico es la técnica de "escenificar canciones emotivas". Este método consiste en incorporar un narración personalizada que se provoca o se crea por medio de la fantasía del niño. Es decir que por medio del uso del juego, se busca la distracción y el humor.

\section{Resultados}

Se trabajó con niños que tienen cáncer, los cuales tenían como principal problemática la depresión y ansiedad. Se planteó una posible opción para lograr mitigar o disminuir los efectos de esta enfermedad, por tal motivo se dio a conocer nuestro proyecto, el cual además de servir como una distracción para los niños, ayuda a disminuir los síntomas que esta trae consigo. Según, González, Guadalupe y Delgado (2009) exponen que las terapias, quimioterapias y administración de medicamentos los ayuda a integrarse más con otros niños que presentan los mismos síntomas y otros factores psicosociales que pueden generar el cáncer. Por tal motivo se implementa un juguete llamado Cars Ecology, el cual contribuye a las terapias y quimioterapias ayudando a ver mejoras en la salud del niño. 


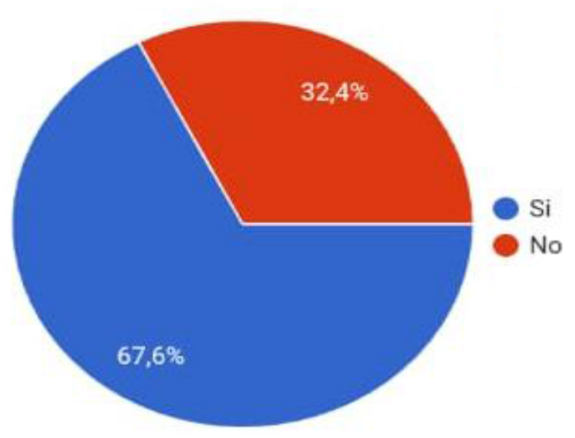

Figura 1. Familiar con antecedente de cáncer. Fuente: elaboración propia.

En la figura 1, podemos observar que en la pregunta contestada por 37 personas, se evidencia que un $67,6 \%$ sí tienen amigos o conocidos con antecedentes de cáncer y un 32,4\% no tienen amigos ni conocidos con antecedentes de cáncer.

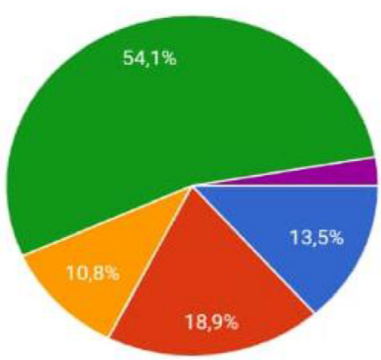

$$
\begin{aligned}
& \text { Escuchar al niño } \\
& \text { que padece esta } \\
& \text { enfermedad } \\
& \text { Recibir } \\
& \text { acompañamiento } \\
& \text { constante del eq... } \\
& \text { Buscar } \\
& \text { acompañamiento } \\
& \text { psicológico } \\
& \text { Disminuir el } \\
& \text { impacto de la en... } \\
& \text { hacerla sentir } \\
& \text { bien emocional... }
\end{aligned}
$$

Figura 2. Acompañamiento niño con cáncer. Fuente: elaboración propia.

En la figura 2, podemos identificar que de un total de 37 personas que contestaron esta pregunta, el $54,1 \%$, consi- dera que disminuir el impacto de la enfermedad es un buen acompañamiento para un niño que tiene cáncer. Sin embargo, un $18,9 \%$ está de acuerdo con que el buen acompañamiento va por parte del equipo médico, un 10,8 \% en buscar acompañamiento psicológico para el niño durante su proceso de enfermedad, un 13,5\% que es mejor escuchar al niño, y una sola persona indica que hacerlo sentir bien emocionalmente y cumplir sus necesidad es un buen acompañamiento.

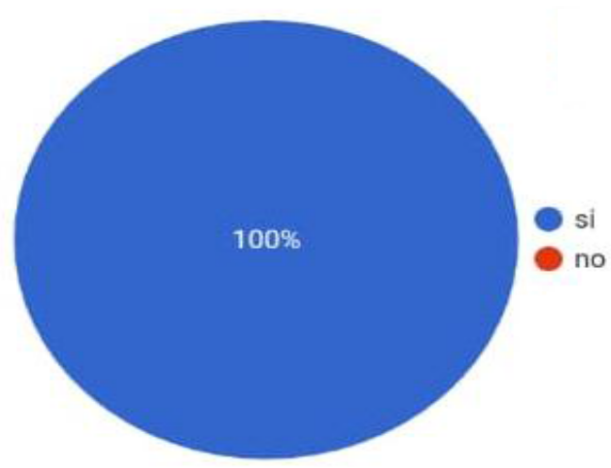

Figura 3. Afectación emocional a un niño con cáncer.

Fuente: elaboración propia.

La figura 3 nos da a conocer que de un total de 37 personas que contestaron la encuesta, todos opinan que el cáncer afecta emocionalmente a un menor de edad. 


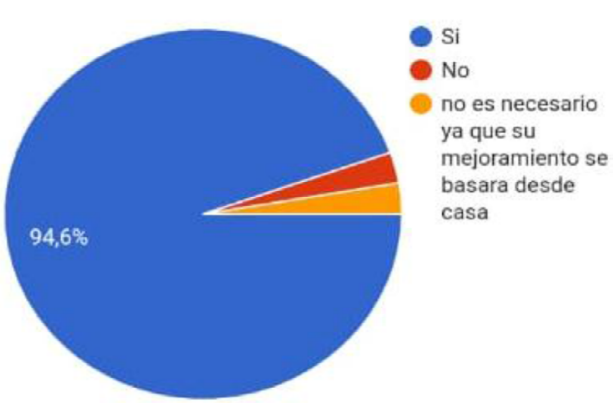

Figura 4. Terapia Cars Ecology.

Fuente: elaboración propia.

En la figura 4 podemos observar que de un total de 37 personas que contestaron, el 94,6 \% sí está de acuerdo en continuar con la terapia de Cars Ecology para cuando se le dé de alta; el 2,7\% contestó que no está de acuerdo en continuar la terapia extrahospitalariamente y el 2,7 \% contestó que no es necesario ya que su mejoramiento se dará desde su casa.

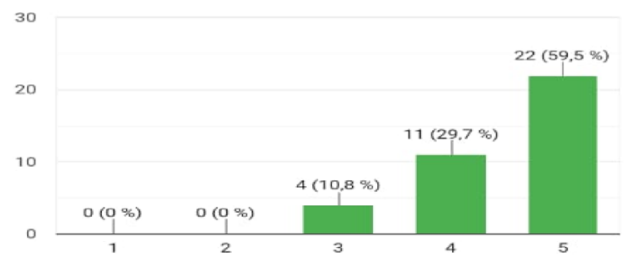

Figura 5. Depresión y estrés.

Fuente: elaboración propia.

En la figura 5 podemos observar los resultados a la pregunta: ¿Considera que la depresión y estrés es causado por el peso que conlleva al dolor extenso de esta enfermedad? Conteste 1 a 5 consi- derando 1 "no es necesario" y 5 "sí es muy necesario". Se ha demostrado que los juguetes ayudan al nivel terapéutico para afrontar las terapias, quimioterapias y otras funcionalidades médicas. De 37 respuesta el (10,8\%) contestó 3 como muy necesario, el (29,7\%) 4 como muy necesario y finalmente el $(59,5 \%)$ le dio un puntaje de 5 como muy necesario.

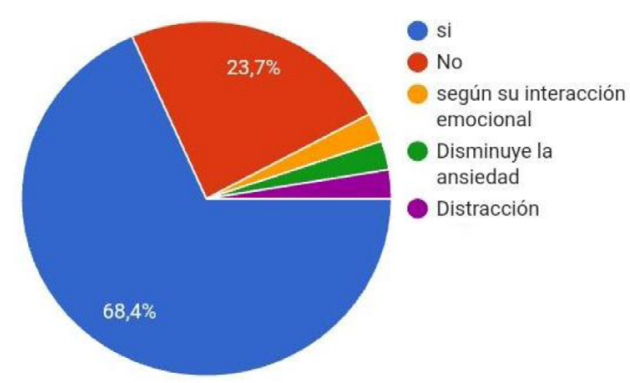

Figura 6. Influencia de los juguetes en un niño con cáncer.

Fuente: elaboración propia.

La figura 6 nos manifiesta la importancia que influyen los juguetes en la vida de un niño con cáncer: el $(68,4$ \%) de 32 personas contestaron sí, el $(23,7 \%)$ no y finalmente un $2,6 \%$ contestó "otras opciones" como "según su interacción emocional”, "disminuye la ansiedad" y "distracción”.

\section{Discusión y conclusiones}

Con los resultados que fueron expuestos en el grupo de trabajo, te- 
niendo en cuenta el marco teórico, la importancia de la depresión, factores psicosociales que representa el cáncer y la felicidad que el niño adquiere por medio de un juguete, llegamos a la conclusión del conocimiento previo de cómo se puede tratar y generar un acompañamiento a quién padece esta enfermedad. Cabe resaltar la importancia de tener un conocimiento acerca de este tema, teniendo claro que los pacientes se encuentran a la expectativa de los cuidados que les vamos a brindar como profesionales de la salud. Aunque también el aporte que vamos a brindar con Cars Ecology se enfoca en disminuir la depresión y estrés causada por los diferentes tratamientos a los cuales son sometidos, identificamos de manera clara que la depresión siempre va a estar en cualquier enfermedad y este carro hecho con latas brindará un tiempo de hospitalización más agradable a los niños.

De Rivas-Molina, Mireles-Pérez, Soto-Padilla, González-Reyes, Barajas-Serrano y Barrera (2015) manifiestan que en el hospital contaba con una población de 1,260 pacientes con diagnóstico de enfermedades hematológicas; de los cuales 222 niños (17\%) estaban diagnosticados de leucemia. Únicamente se estudiaron 46 (21\%) pacientes que cumplieron los criterios de selección (figura 7). En la tabla 1 se describen las características demográficas de los pacientes estudiados. Observamos mayor frecuencia en niños de género masculino. El perfil de la enfermedad se observa sobre todo en niños con LLA principalmente en fase de consolidación. El 9 \% de la población total tuvo algún antecedente familiar de trastornos depresivos. Observamos grandes diferencias en los dos grupos al analizar el tipo de leucemia, la fase de tratamiento ni las recaídas de la enfermedad. En cambio, sí se observan diferencias, con implicación estadística, en el antecedente de depresión familiar. El $95 \%$ de los niños con depresión no tuvieron antecedentes familiares, con una diferencia estadística con un valor de $\mathrm{p}$ $=0.017$.

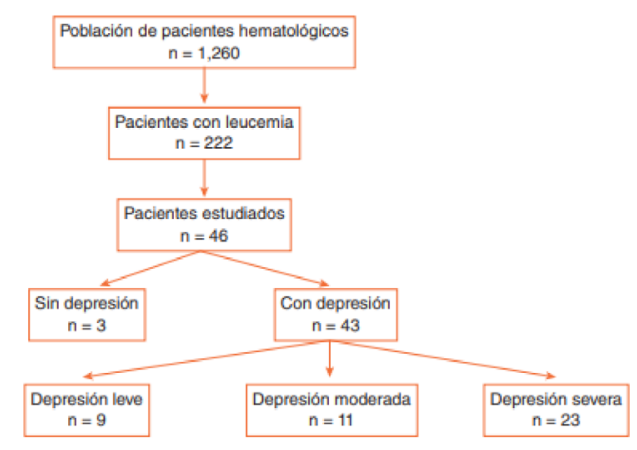

Figura 7. Perfil general del estudio de depresión en niños.

Fuente: Rivas-Molina, et al. (2015). 
Tabla 1. Características clínicas y sociodemográficas de los adolescentes estudiados en fase de tratamiento por leucemia.

\begin{tabular}{|c|c|}
\hline Caracteristica & Valor $(n=46)$ \\
\hline Género, masculino/femenino, $\mathrm{n}(\%)$ & $32(70) / 14(30)$ \\
\hline Edad en anos, mediana (rango) & $8(7-15)$ \\
\hline \multicolumn{2}{|l|}{ Estado civil de los padres } \\
\hline Casados, n (\%) & $41(89)$ \\
\hline Solteros, $n(\%)$ & $1(2)$ \\
\hline Unión libre, $\mathrm{n}(\%)$ & $3(7)$ \\
\hline Viudo, $n(\%)$ & $1(2)$ \\
\hline Antecedente familiar de depresión, $n(\%)$ & $4(9)$ \\
\hline \multicolumn{2}{|l|}{ Tipo de leucemia } \\
\hline LLA, $n(\%)$ & $42(91)$ \\
\hline LMA, $\mathrm{n}(\%)$ & $4(9)$ \\
\hline \multicolumn{2}{|l|}{ Fase de tratamiento } \\
\hline Inducciōn, n (\%) & $12(26)$ \\
\hline Mantenimiento, $\mathrm{n}(\%)$ & $2(4)$ \\
\hline Consalidación, $n(\%)$ & $32(70)$ \\
\hline Paciente con recalidas, $\mathrm{n}(\%)$ & $21(46)$ \\
\hline
\end{tabular}

Fuente: Rivas-Molina et al. (2015).

Teniendo en cuenta la importancia del tema tratado es muy importante el poder llegar a combatir este enemigo silencioso que es la depresión por medio de acciones como el acompañamiento y la participación estrella de nuestro Cars Ecology. Este es una herramienta fundamental para el desarrollo de nuestro trabajo, logrando así intervenciones apropiadas para el acompañamiento del paciente que lo necesite, basándonos en artículos de base científica y desarrollando el paso a paso en grupo, para así entregar un producto final que logre llenar las expectativas de las personas que van a usar nuestro producto para hacer feliz a un niño.

\section{Referencias}

Aquino Martínez, H. E., González, J., Guadalupe, K., y Delgado, O. M. H. (2009). Programa de terapia lúdica orientado a la disminución de las alteraciones del estado de ánimo en niños/as de la tercera infancia que adolecen de enfermedad médica y que tienen un periodo mayor de cuatro días en el hospital nacional de niños Benjamín Bloom. Recuperado de: https://webquery.ujmd.edu.sv/siab/bvirtual/BIBLIOTECA\%20VIRTUAL/TESIS/03/PSI/ ADAP0000341.pdf

Bragado, C. (2009). Funcionamiento psicosocial e intervenciones psicológicas en niños con cáncer. Psicooncología, 6(2/3), 327.

Calvillo, M. E. N. (2004). Depresión en niños con trastorno por déficit de atención con hiperactividad. Revista Reflexiones, 83(1).

Dávila Riega, D. E. L. (2017). Características Clínico-Epidemiológicas y Frecuencia de Depresión y Riesgo de Suicidio en Pacientes con Cáncer en Estado Terminal en el Instituto Regional de Enfermedades Neoplásicas del Sur-Arequipa-2017.

González, H. (2006). Depresión en niños y niñas con cáncer, Facultad de Psicología, Universidad Autónoma de San Luis Potosí, México, Recuperado de: https:// dialnet.unirioja.es/servlet/articulo?codigo $=4794920$

Grau Rubio, C. (2002). Impacto psicosocial del cáncer infantil en la familia. 
Hernández, Y. D. R. G. (2006). Depresión en niños y niñas con cáncer. Actualidades en psicología, 20(107), 22-44.

Medina, V. (2019). Claves para potenciar la felicidad en los niños. Recuperado de: https:// www.guiainfantil.com/blog/771/8-claves-para-potenciar-la-felicidad-en-los-ninos.html

Méndez, F. X., Ortigosa, J. M., y Riquelme, A. (2009). Afrontamiento psicológico de los procedimientos médicos invasivos y dolorosos aplicados para el tratamiento del cáncer infantil y adolescente: la perspectiva cognitivo-conductual. Psicooncología, 6(2), 413-428.

Milagros, L. S. (2017). El valor de las actuaciones pedagógicas en el ámbito hospitalario. Recuperado de: http://hdl.handle. net/10366/136037
Pérez Mipaz, D. E., y Reyes Itas, D. A. (2017). Estresores Hospitalarios en la Adaptación y Recuperación de la salud del Paciente Pediátrico de 5 a 14 Años en el Área de Pediatría del Hospital San Vicente de Paúl Periodo 2015-2016 (Bachelor's thesis). http://repositorio.utn.edu.ec/handle/123456789/6180

Rivas-Molina, N. S., Mireles-Pérez, E. O., Soto-Padilla, J. M., González-Reyes, N. A., Barajas-Serrano, T. L., y Barrera de León, J. C. (2015). Depresión en escolares y adolescentes portadores de leucemia aguda en fase de tratamiento. Gac Med Mex, 151(2), 186-191.

Segura, D. T. (2015). La creación de escenarios fílmicos en el cine musical de Marisol y Joselito Recursos de exaltación del artista1. FRONTERAS reales, 153.

Shapiro, L. E. (2002). La salud emocional de los niños (Vol. 16). Edaf. 